\title{
Agentification of EleCtronic HeALTHCARE RECORD SYSTEMS
}

\author{
SERBANATI, L. \& VAsilateAnU, A.
}

Abstract: Contemporary, most modern electronic healthcare record systems (EHR-S) have service oriented architectures. In the paper our approach to developing an advanced regional EHR-S that supports not only the patient's care process due to individual care providers but also the management of teams in interdisciplinary teambased care processes is presented. Such an EHR-S enables creation in the virtual world of ad hoc organizations of agents that reflect activities for creation, management, and cessation of care providers teams in the real world. Starting from the architecture of an existing, service oriented EHR-S, currently in use, we propose and describe a stepped agentification methodology, able to gradually evolve a conventional EHR-S to an agent-based integration infrastructure, a prototype of an advanced EHR-S.

Key words: Electronic Health Record System, Virtual Health Record, multi-agent system, agentification, virtualization
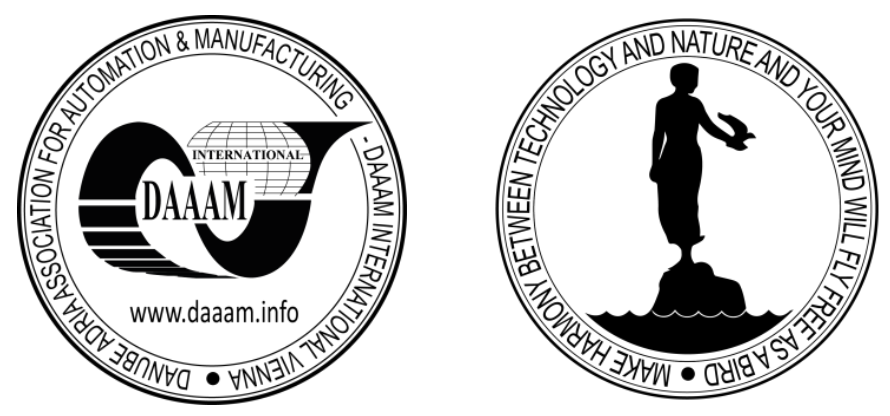

Authors' data: Univ. Em. Prof. Serbanati, L[uca Dan]; Lecturer Dr. Vasilateanu, A[ndrei], University Politehnica of Bucharest, Spl. Independentei 313, 060042, Bucharest, Romania, luca@serbanati.ro, andrei.vasilateanu@upb.ro.

This Publication has to be referred as: Serbanati, L[uca] D[an] \& Vasilateanu, A[ndrei] (2015). Agentification of Electronic Healthcare Record Systems, Chapter 28 in DAAAM International Scientific Book 2015, pp.329-336, B. Katalinic (Ed.), Published by DAAAM International, ISBN 978-3-902734-05-1, ISSN 1726-9687, Vienna, Austria

DOI: $10.2507 /$ daaam.scibook.2015.28 
Serbanati, L. D. \& Vasilateanu, A.: Agentification of Electronic Healthcare Record ...

\section{Introduction}

In the face of the rapid aging of population, increasing prevalence of chronic diseases, and soaring health care costs, the transition towards new models of health care delivery is inevitable. For example, recent trends in healthcare service delivery promote integrated and patient-centric care provided by multidisciplinary teams and multiple care delivery settings along the continuum of a disease. To place the patient at the center of care means also to focus on prevention and wellness, and to attend to the citizen's the physical, mental and spiritual needs. (Haggerty et al., 2003)

Adoption of information technology (IT) in healthcare allows to innovate or reengineer healthcare sectors for promoting the economic sustainability of healthcare services and improving their quality.

Creating an Electronic Health Record System (EHR-S) as an infrastructural platform to enable the access of all major healthcare stakeholders from a jurisdictional area such as a region or country - care providers, patients, payers, and policy makers - to relevant information on the citizens' health state has become a critical foundation for the practice of evidence-based medicine in many countries. EHR-S integrates all clinical applications in the jurisdictional area by allowing them to export own information on the health state of the visited patients in some shared repositories.

At the core of any EHR-S there is the Electronic Health Record (EHR), a longitudinal (lifelong) electronic record of patient health information that are contained in clinical documents released during encounters of patients with their caregivers. A traditional EHR is the point of aggregation and sharing of the health documents related to a patient. To meet the complex demands of evidence-based and patient-centric medicine, just a document management tool as the traditional EHR is insufficient.

We introduced in (Serbanati et al., 2011) the Virtual Health Record (VHR), an evolution of the traditional EHR, aiming to innovate the main processes in health care, research, education and governance for raising the quality of services and improving the health and social wellbeing of citizens. Unlike the traditional EHR, a VHR is based on the relevant discrete data existing in the clinical documents concerning the health of the patient and related to "operational health-related areas" such as: continuity of care, health care, health governance and personnel training, and support for scientific and epidemiological research. Included in the VHR are patients' demographics, diagnosis reports, progress notes, medications, vital signs, immunizations, laboratory data, radiology reports, etc. All this information is partitioned in past encounters and episodes of care, current health state, and care plans in progress.

In our approach VHR is an entity in network (for instance, a cloud computing resource) that contains virtually all the knowledge we have on the health of each individual from the EHR's jurisdictional area. Such an entity must have the ability to record information on clinical events relevant for the individual's health state, analyze and extract from them atomic data which are significant for the individual's health state and ensure a uniform, consistent, and intelligent management of these data, including their aggregation in semantically richer data structures. VHR content is highly structured for fast access. As presented in (Vasilateanu et al., 2011), VHR can also act 
as a security enforcer, using the available data on episodes of care to automatically set access rights for stakeholders.

Our current research regards how better support business scenarios of collaborative, patient-centric care that involve the VHR on one side and multidisciplinary caregiver teams on the other side. The paper presents a step forward in this direction by putting agents to work in a VHR-centered environment created by a Regional EHR-S.

In the Section 2 we briefly describe LUMIR, an existing VHR-based, longitudinal EHR-S. Section 3 proposes and describes a stepped method of agentification of this EHR-S in order to better emulate the behaviour of the multidisciplinary caregiver teams in the real-world health system. Section 4 contains the conclusions concerning the work presented.

\section{A longitudinal, collaborative EHR-S}

The LUMIR project originated from the Italian program for a National ehealth infrastructure as its implementation in Basilicata, a Southern region in Italy. (***TSE, 2006) Initially conceived as a region-wide longitudinal EHR, LUMIR was planned in 2 steps:

- LUMIR 1 addresses the collaboration of general practitioners and other care providers for the care of a patient. For this LUMIR 1 develops and deploys a service oriented infrastructure that accesses a common, distributed repository;

- LUMIR 2 provides a new range of functionalities such as real time monitoring of the relevant health-related events in the life of the patient, their interpretation, and consequent notifications to all interested parties.

- LUMIR 1 is a distributed, component-based software platform focused on semantic interoperability, security, privacy, and reliability issues. (Contenti et al., 2010) It has a layered software-oriented architecture (SOA) as the Fig. 1 shows. On the top layer we find the care providers' clinical applications acting as service consumers. Such a service consumer uses the services in the second layer to notify other applications (thus other caregivers) and the LUMIR 1 system itself of occurrences of relevant health events in its environment. These events are mainly related to the various types of encounters a patient can have with care providers and their organizations. An encounter belongs to some episodes of care the care provider puts in evidence for any health issue of the patient.

To integrate all the local healthcare applications and allow them to communicate with each other, the second layer of the LUMIR1 architecture uses a broker-based, huband-spoke architectural pattern for message transmission and notification dispatching in the distribution network. The network employs high-level services for interoperable message communication, event notification, and document management. The layer promotes a Standard Adapter component as an endpoint for local applications that can be attached to it by specific drivers. Standard Adapter helps to integrate local applications in the SOA by adjusting their peculiarities at the requisites of LUMIR's semantically uniform communication space. The Standard Adapter acts as a boundary agent that takes over information and clinical documents from the caregiver's 
application, verifies and converts the documents in HL7 CDA documents, adds the message header and digital signature, builds the so called "M1 message", encrypts the message content, and finally sends the message across an asynchronous channel to the Broker component. M1 is the HL7 message that boundary agents use to communicate health events to the core agents of the platform.

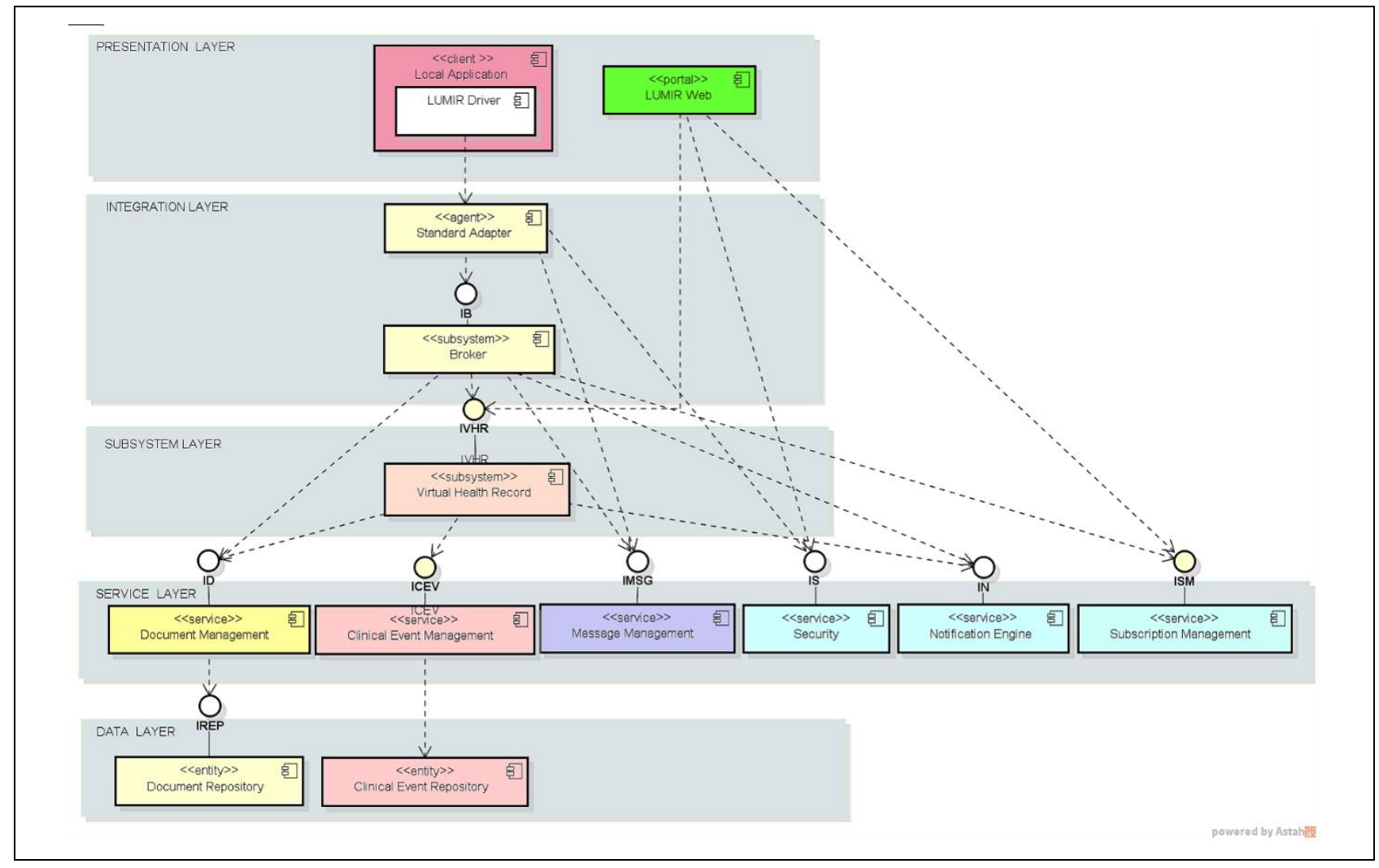

Fig. 1. Lumir 1 Layered Architecture

Presentation layer : Clinical local applications and the EHR-S portal.

Integration layer: Standard Adapters and Broker

Subsystem layer: Virtual Health Record.

Service layer: Document Management, Clinical Event Management, Messaging, Notification, Security, Subscription Management.

Data layer: Document Repository and Clinical EventRepository.

Broker is a multifunctional component that decouples the destinations of the information in the M1 message from the sender and maintains central control over the flow of messages.

VHR is the key subsystem of the EHR-S that exposes specialized, innovative services as we presented before. Other subsystems such as PHR could be added, too.

VHR and Broker use the following services in the subsequent layer:

- Document Management service registers/stores the M1-attached clinical documents in an ebXML registry/repository, and

- Notification service publishes the health event in the VHR, and, according to the event topic and a notification policy, dispatches notification messages, the so called M2 messages, to all destinations that subscribed for the message topic. M2 message 
contains information on the health event and on clinical documents attached to the M1 message;

- Security service assures authentication, authorization, integrity and confidentiality of communications with the environment and inside the EHR-S.

- Experiments with LUMIR 1 demonstrated the EHR-S vocation of integrator of the Regional health information system and this is why LUMIR 2 was proposed as the next step of the project. The role of LUMIR 2 is to enrich the LUMIR 1 service inventory with new, more intelligent services that enable the platform to support new, innovative business use cases including those based on creation and management of virtual teams of caregivers acting as decision-support in medical activities.

\section{Method for developping an EHR-S with agent organizations}

According to our challenging objective, in order to support a patient-centric view on healthcare where ad hoc created teams of caregivers work to care their patients, LUMIR 2 should promote a seamless osmosis of information between the Regional health system in the real world and a multi-agent, computer-based system in the digital world. For this, the computer-based system should "mirror" the real-world one by replicating its real-world resources and behavior in the virtual world. In this operation of "virtualization" the shift to multi-agent systems (MAS) is fully justified by the recurrent MAS features that match situations occurring in the real-world medical settings: delegation of responsibility, re-allocation of tasks, need to consider a large variety of user concerns and problems, planning the collaborative work, think and work in open spaces, etc. (Villar, 2008)

Due to the reasons stated above, usage of MAS in healthcare settings has been a target for a number of research projects. As described in (Isern, 2010) such projects were mainly research-oriented, lacking full scale deployment and validation. The main causes for the limited results is stated to be the lack of involvement of the stakeholders in all the lifecycle of application development, from requirements analysis to implementation, and the poor reuse of existing legacy systems. These considerations do not apply to LUMIR 2 as it is based on a region-wide EHR-S and, being the second phase of a running project, communication with the stakeholders is continuous. LUMIR 2 uses the MAS paradigm to follow an organizational approach where the stakeholders involved in the care process are represented by virtual entities cooperating in a digital environment in order to exchange relevant and operational knowledge. (Wooldridge, 2000)

Because LUMIR 1 is in use, the transition must be seamless for the end-users; they must not perceive any performance downgrade, downtime or feature disappearance. This is why in order to mitigate the risks we chose a stepped "agentification" of the existing software system. "Agentification" corresponds to a progressive evolution of the software architecture style from a component-based SOA style towards a MAS by a gradually increase the autonomy of the software components that should dispose of enough contextual knowledge to autonomously make informed decisions. 
As Fig. 2 shows, the "agentification" process was partitioned in three main stages. In the first stage transition of some software components towards agents is planned. For instance, the Adapter component, currently responsible for integrating the caregivers' legacy applications in the LUMIR space, was promoted to represent the caregiver in virtual. To the LUMIR 1 requirements for Adapter (generation of M1 messages and reception of M2 notifications) we added requirements needed to support workflows of collaboration between caregivers, each of them with her/his profile. From these requirements we extracted the roles of these evolved boundary agents in LUMIR 2 as autonomous, cohesive and collaborating entities with clear purposes and behavior.

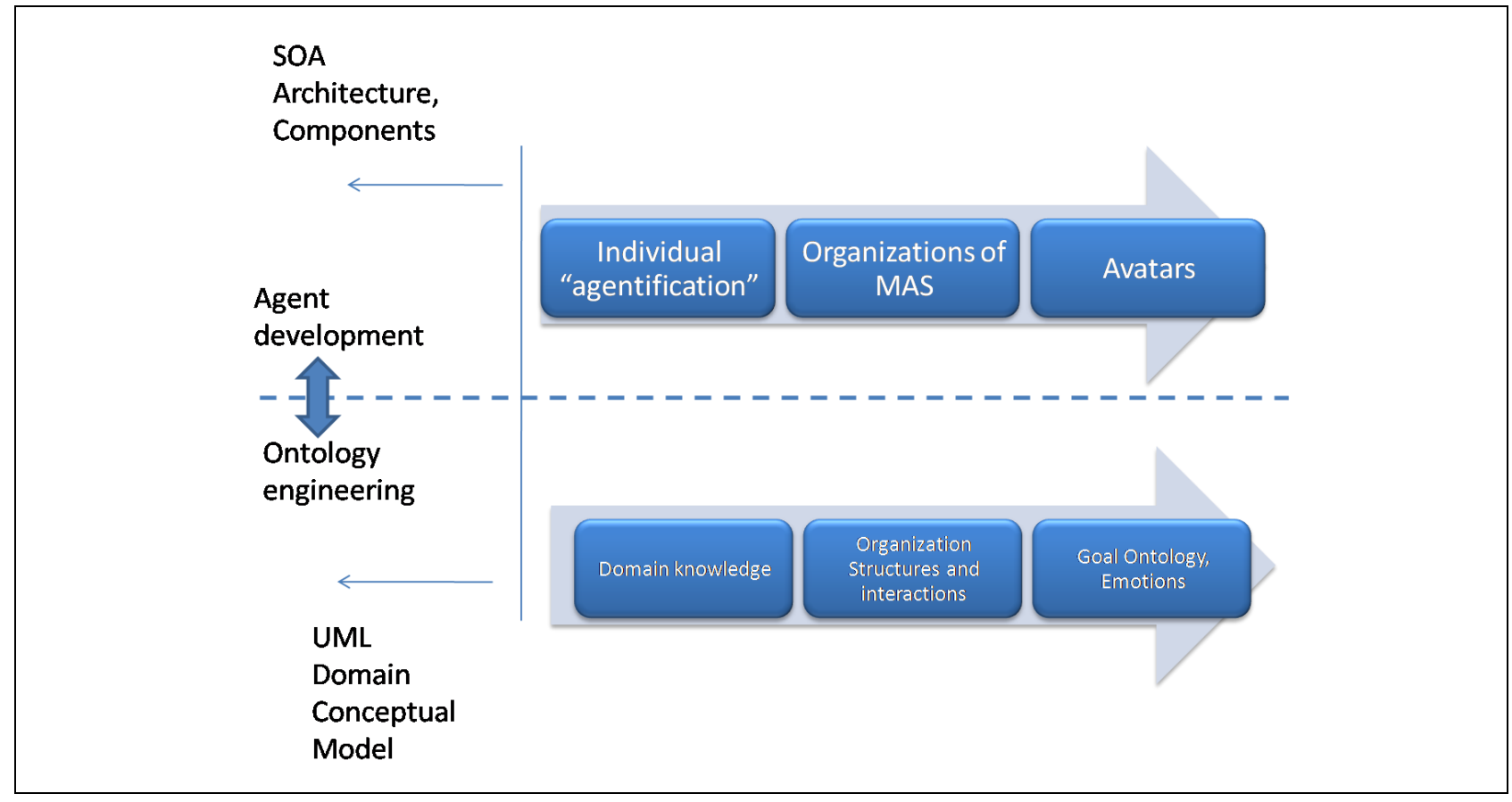

Fig. 2. "Agentification" process

Since the MAS paradigm entails exchange of messages between agents, semantics of messages should be properly captured in ontologies and semantic rules, a parallel task in incrementally designing our MAS is to engineer the field's ontology. LUMIR ontology engineering is planned to be obtained into three partially overlapping phases, closely coordinated with the agent development.

To the first phase of our agent development method corresponds the first phase of the ontology engineering: domain knowledge capturing. We create a coherent ontology necessary to translate enriched M1 and M2 messages by adding new concepts from medical ontologies, to our LUMIR 1 conceptual model. The resulting ontology is roughly composed of two dichotomic parts: endurant and perdurant clinical concepts. The agents we create in this stage can freely communicate with the VHR, itself controlled by a centrally located, complex agent.

In the second stage of our development method we concentrate on supporting multi-disciplinary teams by formalizing the organizational structures getting together doctors of different specialties. We consider an organization as a collectivity of agents exhibiting some explicitly specified social structures and oriented to the pursuit of a set of objectives/goals originating from its setting in a given environment. (Dignum, 
2009) (Vasilateanu, 2013) Organizations can be static or dynamic. Static organizations have their established status-quo in their business, for instance organizations corresponding to regional or local medical institutions like hospitals. To create a virtual organization that mirrors a static organization we only have to map the relevant roles in the real organization to the virtual ones.

Dynamic organizations are created ad-hoc due to a change in the care process environment. To create a virtual care team that mirrors a dynamic organization of caregivers we need a flexible framework allowing a collectivity of agents to spawn, change or dissolve at runtime with roles created on the fly. We introduce the Virtual Healthcare Organization Manager (VHOM), an agent acting as both a policy enforcer in the organization (DeLoach, 2002) and a facilitator, supporting or inhibiting the spawning of individual agents. VHOM "weaves the fabric" of the virtual organization, superimposing organizational patterns and structures on the collection of managed agents. During this stage the ontology is enriched with interaction protocols, roles, negotiation, and mediation knowledge.

In the third stage of the development method we make steps toward contextaware, goal-oriented agents, actively pursuing their stakeholders' interests and concerns in the digital environment by continuous adaptation. (Rentea et al., 2011) We call these agents "avatars", that is "digital" reincarnations of their corresponding stakeholders in the digital environment that act proactively for the fulfillment of their users` concerns in the digital environment. (Serbanati et al., 2011) To represent as accurately as possible in the avatar's universe the complex socio-economic interactions existing in the health system, we need to first identify then formalize in a ad hoc goal ontology the goals of each stakeholder as expressed by her/his external interactions but also influenced by her/his interior needs and concerns.

The internal structure of the agents in the LUMIR 2 MAS is component-oriented. When we want to create a new virtual team, reliable factories for each agent type, themselves agents, create new instances of needed agents by combining off-the-shelf standardized software components. (Ricci et al., 2006) While such an approach has a very promising chance of wide-spread adoption, due to the reasons stated at the start of the section - upgrade of an existing functioning solution, stakeholder implication - we must also consider the threats to its success. The main barrier is non-technical, it is the care provider's resistance to new solutions, especially ones with a more proactive role in the care process, which can even change the care workflow. In order to counter that we stress out that our solution is just a decision support tool, the care provider still has the final decision and total control.

\section{Conclusion}

Information systems in a patient-centric health system should be designed to support interoperability between the caregivers' clinical applications and their cooperation in complex care workflows in a context-aware environment. To this aim more expressive and adaptive paradigms are needed. The paper presents how an experience in developing an EHR-S based on a longitudinal VHR as a service-oriented cloud resource allowed us to gain an informed view on supporting complex business 
Serbanati, L. D. \& Vasilateanu, A.: Agentification of Electronic Healthcare Record ...

processes in advanced healthcare systems with agent-based infrastructures able to recreate a more natural representation of healthcare reality in the digital space.

\section{References}

Contenti, M.; Mercurio, G.; Ricci, F. L., \& Serbanati, L. D. (2010). The LUMIR Project: Developing the GP's Network Pilot Program in the Basilicata Region, in Information Systems: People, Organizations, Institutions, and Technologies, Springer, pp. $255-263$

DeLoach, S. (2002). Modeling organizational rules in the multi-agent systems engineering methodology, Advances in Artificial Intelligence, pp. 1-15

Dignum, V. (ed.) (2009). Handbook of Research on Multi-Agent Systems: Semantics and Dynamics of Organizational Models, Information Science Reference

Haggerty, J. L.; Reid, R. J., Freeman, G. K., Starfield, B. H. \& Adair, C. E. (2003). Continuity of care: a multidisciplinary review, British Medical Journal, vol 327, pp. 12-19

Isern, D. ; Sánchez, D. \& Moreno, A. (2010). Agents applied in health care: A review, International Journal of Medical Informatics, 79.3, pp. 145-166

Rentea, V., Vasilateanu, A. \& Serbanati, L. (2011). Supporting Adaptability in AgentBased Digital Healthcare Ecosystem, Proceedings of The Third International Conference on Advanced Cognitive Technologies and Applications, Rome, September 2011

Ricci, A., Viroli, M. \& Omicini, A. (2006). CArtAgO: An Infrastructure for Engineering Computational Environments in MAS, 3rd Inter. Workshop Environments for Multi-Agent Systems, E4MAS, 2006

Serbanati, L.D., Ricci, F.L., Mercurio, G. \& Vasilateanu, A. (2011). Steps Towards a Digital Health Ecosystem, Journal of Biomedical Informatics, doi: 10.1016/j.jbi.2011.02.011

Vasilateanu, A., \& Serbanati, L.D. (2011). E-Health Workflow-based Authorization using an Agent-oriented Virtual Health Care Record, Proceedings of Proceedings of the 3rd International Conference on Agents and Artificial Intelligence, Rome, January 2011

Vasilateanu, A. ; Margarit, A. \& Radulescu, A.C. (2013). Virtual Agent organizations Supporting disease specific communities. Proceedings of E-Health and Bioengineering Conference, Iasi, November 2013

Villar, A.; Federici A., \& Annicchiarico, R., (2008). Agents and Healthcare: A Glance to the Future, Birkhauser Science, pp. 141-148

Wooldridge, M.J. (2000): Multiagent Systems: A Modern Approach to Distributed Artificial Intelligence, MA: MIT Press

*** TSE, Italian National Board for eHealth (2006). Architectural Strategy for eHealth ***http://www.sanitaelettronica.gov.it/xoops/modules/docmanager/index.php?curent _dir=39 TSE-IBSE-Strategia_architetturale-v01.00-DEF.pdf, (in Italian), 2006 